\title{
Faixas críticas de concentrações de nutrientes e avaliação do estado nutricional de cafeeiros em quatro regiões de Minas Gerais ${ }^{(1)}$
}

\author{
Herminia Emilia Prieto Martinez ${ }^{(2)}$, June Faria Scherrer Menezes ${ }^{(3)}$, Ronessa Bartolomeu de Souza ${ }^{(4)}$, \\ Víctor Hugo Alvarez Venegas ${ }^{(5)}$ e Paulo Tácito Gontijo Guimarães ${ }^{(6)}$
}

Resumo - Os objetivos deste trabalho foram definir as faixas críticas de concentração de nutrientes e avaliar o estado nutricional das lavouras cafeeiras de alta, média e baixa produtividade em quatro regiões do Estado de Minas Gerais. Foram coletadas folhas em 312 unidades amostrais de 168 lavouras, tanto no ano de alta produtividade quanto no de baixa produtividade, conforme o ciclo bienal do cafeeiro. Nas folhas foram determinadas as concentrações de macronutrientes e dos micronutrientes $\mathrm{Cu}, \mathrm{Fe}, \mathrm{Zn}$, Mn e boro. Com os resultados das análises foliares, estabeleceram-se as faixas críticas das concentrações dos nutrientes no ano de baixa e alta produtividade, em cada região estudada, utilizando como referência lavouras de alta produtividade (mais de $1.800 \mathrm{~kg} / \mathrm{ha}$ de café beneficiado na média do biênio). Realizou-se ainda o diagnóstico do estado nutricional das lavouras nas regiões em estudo determinandose as freqüências de nutrientes em níveis deficiente, adequado e excessivo. As faixas críticas não diferiram entre os anos amostrados, tampouco entre as regiões em estudo. Nas regiões amostradas predominam problemas com micronutrientes.

Termos para indexação: Coffea arabica, diagnose foliar, distúrbio nutricional, nível crítico.

\section{Critical nutrient ranges and evaluation of nutritional status in coffee-tree plantations of Minas Gerais}

\begin{abstract}
The objectives of this work were to define the critical nutrients ranges and evaluating the nutritional state of coffee-tree plantations with low, intermediate and high productivities in four areas of the State of Minas Gerais. Leaves of 312 sample units of 168 farms were collected in two consecutive years, one of high productivity and another of low productivity, according to the biennial cycle of the coffee-tree. In the leaves all the macronutrients and the micronutrients $\mathrm{Cu}, \mathrm{Fe}, \mathrm{Zn}, \mathrm{Mn}$ and $\mathrm{B}$ were analyzed. With the results of the analyses the critical ranges of the nutrients in the years of low and high productivity and for each studied area were calculated, using as reference farms of high productivity (more than 1,800 kg/ha of green coffee in the average of two consecutive years). The diagnosis of the nutritional state of the farms in the studying areas was made through the frequencies of nutrients in deficient, appropriate and excessive levels. The obtained critical ranges did not differ either between the sampled years or among the areas. In the studied area nutritional problems with micronutrients were predominant.
\end{abstract}

Index terms: Coffea arabica, foliar diagnosis, nutritional disorders, critical level.

(1) Aceito para publicação em 13 de março de 2003 . Extraído da tese de doutorado apresentada pelo segundo autor à Universidade Federal de Viçosa (UFV), Viçosa, MG. Financiado pela Fapemig e pelo CBP\&D/Café.

(2) UFV, Dep. de Fitotecnia, Avenida P. H. Rolfs, s/no CEP 36571-000 Viçosa, MG. Bolsista do CNPq. E-mail: herminia@ufv.br

(3) Universidade de Rio Verde, Caixa Postal 104, CEP 75901-970 Rio Verde, GO. E-mail: june@fesurv.br

(4) UFV, Dep. de Fitotecnia. Bolsista do CBP\&D/Café. E-mail: rbs@solos.ufv.br

(5) UFV, Dep. de Solos. Bolsista do CNPq. E-mail: vhav@ufv.br

(6) Empresa de Pesquisa Agropecuária de Minas Gerais, Centro Tecnológico do Sul de Minas, Caixa Postal 176, CEP 37200-000 Lavras, MG. E-mail: paulotgg@ufla.br, epamig@ufla.br

\section{Introdução}

A cafeicultura é de grande importância na economia Brasileira e constitui-se numa das principais atividades agrícolas do Estado de Minas Gerais. Embora com a maior área cultivada ( $750 \mathrm{mil} \mathrm{ha)} \mathrm{e} \mathrm{a} \mathrm{maior}$ produção (13,9 milhões de sacas de café beneficiado), segundo Oliveira \& Alves (2001), grande parte das lavouras cafeeiras do Estado possui baixa produtividade (menos de $900 \mathrm{~kg} / \mathrm{ha} / \mathrm{ano}$ de café beneficiado), principalmente pela falta de informações sobre manejo nutricional da cultura. 
Para o estabelecimento de um programa apropriado de adubação é necessário identificar os principais problemas inerentes à nutrição da planta e, posteriormente, determinar quais os nutrientes são limitantes, suas quantidades, épocas e formas de aplicação corretas.

As análises químicas do solo e da planta auxiliam no diagnóstico do estado nutricional das culturas, porém apresentam limitações. A análise do solo caracteriza apenas a disponibilidade de nutrientes, ao passo que a análise de tecidos fornece indicações sobre o estado nutricional da planta. Resultados de análises de tecidos podem ser interpretados após comparações com padrões obtidos de populações de plantas altamente produtivas, da mesma espécie e variedade (Malavolta et al., 1997).

A composição mineral dos tecidos vegetais pode ser influenciada por uma série de fatores pertinentes à própria planta e ao ambiente: espécie, variedade ou porta-enxerto, estádio vegetativo e idade da planta, distribuição, volume e eficiência do sistema radicular, produção pendente, variações climáticas, disponibilidade de água e nutrientes no solo, estado fitossanitário da planta, tipo e manejo do solo e interações entre nutrientes. Assim, a obtenção de padrões apropriados a partir da análise de tecidos é de fundamental importância (Ingestad \& Agren, 1995; Martinez et al., 1999).

Entre os critérios para interpretar os resultados da análise química, o nível crítico ainda é o mais utilizado; acrescido da produção de massa seca foi superior ao DRIS no diagnóstico do estado nutricional do milho (Soltampour et al., 1995).

As maiores vantagens do uso de níveis críticos são a facilidade de interpretação dos resultados e a independência entre os níveis (a concentração de um nutriente não afeta a classificação do outro). Entretanto, apresenta como desvantagens a impossibilidade de determinar o grau da deficiência ou do excesso e, ainda, a limitação em identificar qual o nutriente mais problemático, quando mais de um nutriente é limitante (Baldock \& Schulte, 1996; Lucena, 1997; Martinez et al., 1999).

O nível crítico de um determinado nutriente na planta é definido como o valor da concentração que separa a zona de deficiência da zona de suficiência. Acima dele, a probabilidade de haver aumento na produção pela adição do nutriente é baixa; abaixo, a taxa de crescimento, a produção e a qualidade diminuem significativamente (Lagatu \& Maume, 1934, citados por Smith, 1988). Para aumentar a flexibilidade da diagnose, considera-se uma faixa e não um único valor crítico (Lucena, 1997; Martinez et al., 1999, 2000). Malavolta \& Cruz (1971) definiram a concentração crítica como a faixa de concentração de um elemento na folha abaixo da qual a produção é limitada e acima da qual a adubação não é econômica.

Apesar de serem encontrados na literatura níveis críticos dos nutrientes na folha do cafeeiro (Willson, 1985; Reuter \& Robinson, 1988; Jones Junior et al., 1991; Malavolta, 1993; Mills \& Jones Junior, 1996; Malavolta et al., 1997; Matiello, 1997), ao serem estabelecidos, não foram consideradas as variações regionais, sendo necessário estabelecer padrões próprios para cada região ou Estado, para uma avaliação nutricional mais precisa.

Os objetivos deste trabalho foram definir as faixas críticas de concentração dos nutrientes e avaliar o estado nutricional de lavouras cafeeiras de alta, média e baixa produtividades em quatro regiões do Estado de Minas Gerais.

\section{Material e Métodos}

Nos anos agrícolas de 96/97, 97/98 e 98/99 foram coletadas amostras de folhas de cafeeiros em talhões homogêneos de 0,5 a 1,0 ha demarcados em lavouras de cinco a nove anos de idade e com população variando entre 3.000 e 5.000 plantas/ha, nos municípios mineiros de Manhuaçu, Patrocínio, Guaxupé - São Sebastião do Paraíso e Viçosa. Foram coletados o 3o e 4o pares de folhas a contar do ápice do ramo na altura mediana da planta, em 20 plantas/talhão, na fase de chumbinho. Avaliou-se a produtividade dos talhões amostrados e aplicou-se questionário complementar a respeito dos tratos culturais e adubações realizadas em cada talhão. Nas folhas coletadas foram determinadas as concentrações de macronutrientes e micronutrientes. Trabalhou-se com 168 lavouras, sendo 41 de Viçosa, 36 de Manhuaçu, 44 de Patrocínio e 47 de Guaxupé e São Sebastião do Paraíso. Utilizaram-se as lavouras com alta produtividade (mais de $1.800 \mathrm{~kg} / \mathrm{ha}$ de café beneficiado na média de dois anos consecutivos) para calcular as faixas críticas de macronutrientes e micronutrientes.

Com os resultados das análises foliares, foram determinados, nos anos de alta e baixa produtividade, a média $(\bar{y}), o$ desvio-padrão da média $\left(\mathrm{S}_{\bar{y}}\right)$ e o coeficiente de variação $(\mathrm{CV})$ 
das concentrações de cada nutriente nas folhas das plantas, calculando-se, a partir destes, a faixa crítica $(\mathrm{FC})$ : $\mathrm{FC}=\overline{\mathrm{y}} \pm \mathrm{kS} \overline{\mathrm{y}}$ em que: $\bar{y}$ é a média da concentração do nutriente; $\mathrm{S} \bar{y}$ (desvio-padrão da média) $=\mathrm{S} / \sqrt{\mathrm{r}}$, em que $\mathrm{r}=1 ; \mathrm{k}$ é o fator de correção para evitar faixas críticas muito amplas.

Os valores de $\mathrm{k}$ foram estabelecidos em razão do $\mathrm{CV}$, da seguinte forma:

$\mathrm{k}=1,0$ para os nutrientes cujo $\mathrm{CV}$ foi menor que $20 \%$; $\mathrm{k}=0,8$ para os nutrientes cujo CV variou de $20 \%$ a $40 \%$; $\mathrm{k}=0,6$ para os nutrientes cujo CV variou de $40 \%$ a $80 \%$; e $\mathrm{k}=0,4$ para os nutrientes cujo CV foi maior que $80 \%$.

Nas comparações entre as FC obtidas nos anos de alta e de baixa produtividade foram calculados intervalos de confiança (IC):

$\mathrm{IC}=\overline{\mathrm{y}} \pm \mathrm{t} \alpha \cdot \mathrm{S} \overline{\mathrm{y}}$

em que: $\bar{y}$ é a média da concentração do nutriente; $\mathrm{S} \bar{y}=$ desvio-padrão da média; t $\alpha$ é o valor de t bilateral, a $10 \%$ de probabilidade, com n-1 número de lavouras de alta produtividade.

Os intervalos de confiança com valores negativos foram substituídos por zero.

Para cada nutriente, faixas críticas ajustadas que não apresentaram pontos de interseção foram consideradas diferentes entre si.

Como as diferenças encontradas entre as faixas críticas em anos de alta e baixa produtividade foram mínimas, os dados dos dois anos consecutivos foram reunidos e utilizados para novo cálculo de faixas críticas, seguindo-se o método descrito anteriormente. De forma similar, a comparação entre as faixas críticas, nas regiões estudadas, foi realizada com base nos intervalos de confiança.

Utilizando-se as faixas críticas para cada nutriente, avaliou-se o estado nutricional dos cafeeiros amostrados, dentro de cada região específica, nos anos de alta e baixa produtividade, separando-se as lavouras em três classes de produtividade: alta (mais de $1.800 \mathrm{~kg} / \mathrm{ha} /$ ano de café beneficiado, na média de dois anos), média (de 900 a $1.800 \mathrm{~kg} / \mathrm{ha} /$ ano de café beneficiado, na média de dois anos) e baixa (menos de $900 \mathrm{~kg} / \mathrm{ha} /$ ano de café beneficiado, na média de dois anos).

As concentrações dos nutrientes nas folhas de cada lavoura foram classificadas em excessivas, adequadas e deficientes. Posteriormente, foram calculadas as freqüências de lavouras com concentrações foliares excessivas, adequadas e deficientes, em relação a cada nutriente analisado e em cada região. Para indicar os principais problemas nutricionais, consideraram-se os nutrientes cujos teores não estavam dentro das faixas críticas calculadas em mais que $50 \%$ das lavouras amostradas em cada região.

\section{Resultados e Discussão}

Apenas no Município de Manhuaçu a faixa crítica de $\mathrm{B}$ no ano de alta produção foi inferior à obtida no ano de baixa produção (Tabela 1). Faixas críticas semelhantes nos anos de alta e baixa produtividade (Tabela 1) indicam que na época em que as folhas foram amostradas os frutos ainda não apresentavam acentuada atividade de dreno e confirmam que a fase compreendida entre o florescimento e a primeira fase de expansão rápida dos frutos é indicada para as avaliações do estado nutricional do cafeeiro. Leece \& Ende (1975), Kotur \& Singh (1993) e Drossopoulos et al. (1996), recomendam que se amostre as folhas em períodos e posições na planta em que sejam mínimas as variações das concentrações de nutrientes em virtude da flutuação estacional.

De modo geral, as faixas críticas obtidas no presente trabalho estão próximas das encontradas na literatura quanto a N, Ca e enxofre. São superiores em relação ao $\mathrm{Cu}$ e $\mathrm{Mn}$, e inferiores em relação ao $\mathrm{Fe}$ e ao $\mathrm{Zn}$ (Tabela 2). Observa-se que a regionalização das normas propiciou reduções nas amplitudes das faixas de suficiência, especialmente quanto ao $\mathrm{P}, \mathrm{S}$ e micronutrientes, o que permite obter diagnóstico mais preciso (Tabelas 1 e 2).

Em Manhuaçu, no ano de alta produção, nas lavouras de alta produtividade predominaram as deficiências de B e manganês. Nesse mesmo ano, as lavouras de média produtividade apresentaram deficiências de B e fósforo. Nas lavouras de baixa produtividade ocorreram excessos de S, Fe e manganês. No ano de produtividade alta, houve deficiências de $\mathrm{P}, \mathrm{Zn}$ e $\mathrm{Cu}$ em lavouras de média produtividade, e deficiências de $\mathrm{N}$ e $\mathrm{Cu}$ nas de baixa produtividade (Figura 1). No ano de baixa produção, houve concentrações excessivas de B em lavouras de alta, média e baixa produtividades. Constataram-se ainda teores excessivos de $\mathrm{K}$ nas lavouras de média produtividade, e de $\mathrm{K}, \mathrm{Mg}$ e Mn nas de baixa produtividade (Figura 1).

Concentrações limitantes de Mn em tecidos foliares, provavelmente, advêm de lavouras cultivadas em solos com baixos conteúdos deste elemento, ou de solos com pH elevado (acima de 5,5), o qual limita sua disponibilidade para as plantas. Assim, a deficiência de Mn pode estar associada à aplicação 


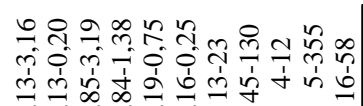
至

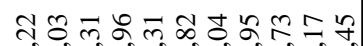

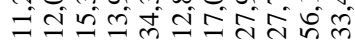

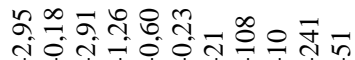

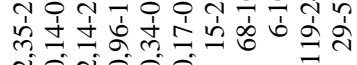

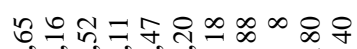
तOnनOO

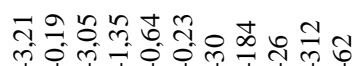

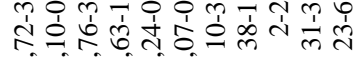
तो $0^{\circ} 00^{\circ}$

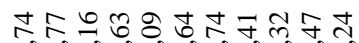

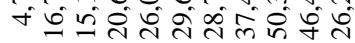
은든 은

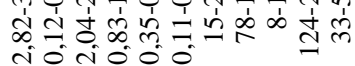

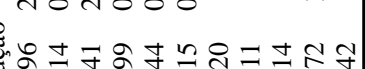

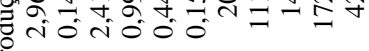
니잉

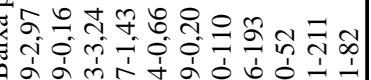

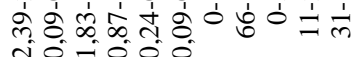
(1)

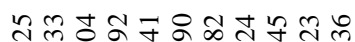
ऊ.

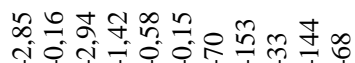

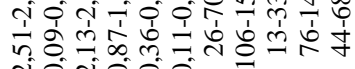
$\infty m m i n \infty m 00$

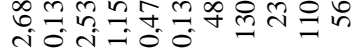
을 $\vec{F} F$

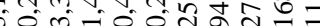

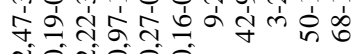
ion

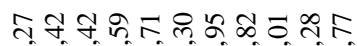

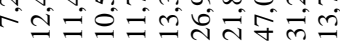
\%

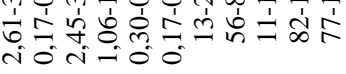

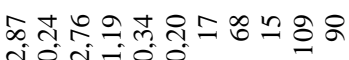

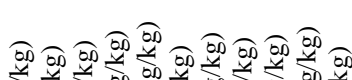

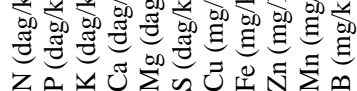

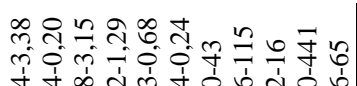

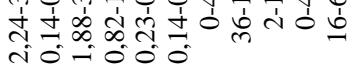
우송

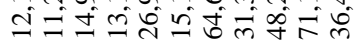

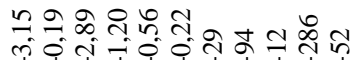

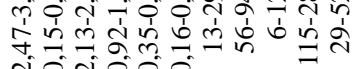

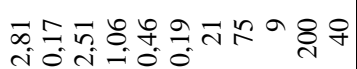

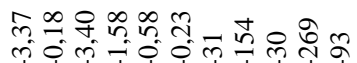

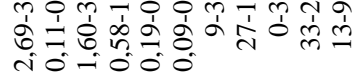

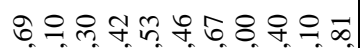

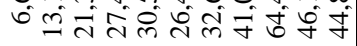

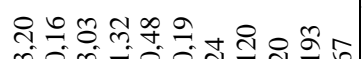
m. तOन000

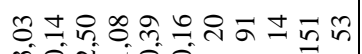
.

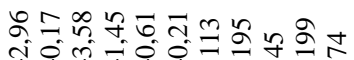

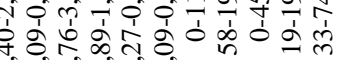
Non 000

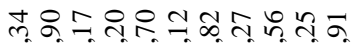

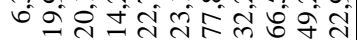

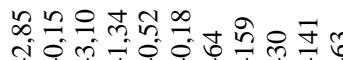

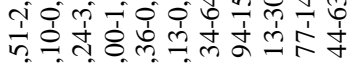

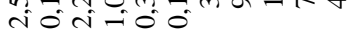

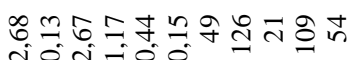
m

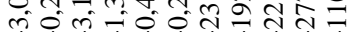

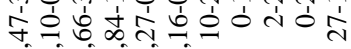
กิ

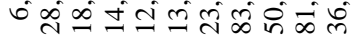

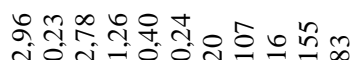

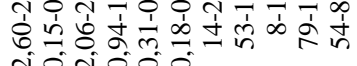
ióñón

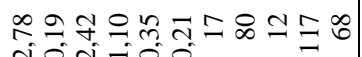

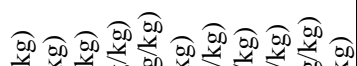

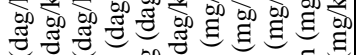

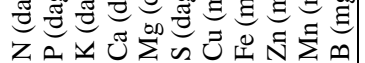


de quantidades excessivas de calcário no ano em que havia perspectivas de produção elevada. Deficiências de $\mathrm{B}$ e $\mathrm{P}$ podem também decorrer de $\mathrm{pH}$ elevado. No caso do $\mathrm{P}$, a elevação do $\mathrm{pH}$ pode resultar em predominância de íons $\mathrm{HPO}_{4}{ }^{2-} \mathrm{e}^{\mathrm{PO}_{4}{ }^{3-} \text { na solu- }}$ ção do solo, os quais podem formar fosfatos bicálcicos e tricálcicos insolúveis. O íon borato, por sua vez, tem sua adsorção a sesquióxidos e minerais de argila aumentada com o aumento do $\mathrm{pH}$, o que limita sua disponibilidade para as plantas (Loomis \& Durst, 1992). Em culturas perenes é comum ocorrer pH elevado quando não se considera a profundidade efetiva em que o corretivo atuará $(5-10 \mathrm{~cm})$ no cálculo da dose de corretivo a ser aplicada. Embora o sistema radicular do cafeeiro seja profundo, como a maior parte de suas raízes absorventes concentra-se na estreita camada superficial, os efeitos do $\mathrm{pH}$ elevado nessa camada podem causar deficiências.

Por sua vez, os excessos de Fe e Mn observados nas lavouras de baixa produtividade indicam condição de elevada acidez e conseqüentemente de solos não corrigidos, ou de uso de quantidades insuficientes de corretivo. Chama atenção a diferença observada em dois anos consecutivos quanto às concentrações de B, que num primeiro momento se apresentaram insuficientes, para passar à condição de excesso no ano subseqüente. É possível que a constatação de uma situação de carência tenha levado a adubações corretivas em excesso. A deficiência de $\mathrm{Cu}$ tem se acentuado em lavouras em que fungicidas cúpricos não fazem parte do programa de combate à ferrugem.
No ano de alta produção, no grupo das lavouras de alta produtividade, em relação aos micronutrientes $\mathrm{Mn}, \mathrm{Cu}$ e $\mathrm{Zn}$ houve mais de $50 \%$ de lavouras com concentrações fora da faixa crítica proposta para Patrocínio (Figura 2). No ano de baixa produção, os problemas detectados foram os mesmos e de mesma magnitude com $\mathrm{Zn}$ e $\mathrm{Cu}$, tornando-se menos acentuados com o manganês. Neste ano houve lavouras com concentrações de $\mathrm{S}$ fora da faixa crítica proposta, predominando a deficiência desse elemento (Figura 2). O uso de doses mais baixas de adubo nitrogenado (sulfato de amônio) contendo S no ano de baixa produção possivelmente tenha promovido essa carência.

Nas lavouras de média produtividade, no ano de alta produção observaram-se porcentuais elevados de problemas com $\mathrm{Cu}, \mathrm{Fe}, \mathrm{Zn}$ e boro. Em relação ao $\mathrm{Zn}$ e B predominaram os excessos e ao $\mathrm{Cu}$ e Fe, as deficiências. No ano de baixa produção, alto porcentual de lavouras apresentou concentrações inadequadas de $\mathrm{Cu}, \mathrm{Zn}$, $\mathrm{K}$ e manganês. Em termos de $\mathrm{Cu}, \mathrm{Mn}$ e Zn, as deficiências e excessos apresentaram proporções similares, e em relação ao K, predominou a deficiência (Figura 2).

A maioria das lavouras de baixa produtividade da região de Patrocínio apresentou concentrações inadequadas de todos os micronutrientes avaliados tanto no ano de alta como no de baixa produção com deficiências de $\mathrm{Cu}, \mathrm{Mn}$ e $\mathrm{Fe}$ em ambos os anos. É possível que o manejo da calagem nessas lavouras tenha sido o responsável por essas carências. Quanto ao B, verificou-se excesso no ano de alta e deficiên-

Tabela 2. Faixas críticas das concentrações de nutrientes em folhas de cafeeiro, segundo alguns autores.

\begin{tabular}{lcccccc}
\hline \multirow{2}{*}{ Nutriente } & \multicolumn{7}{c}{ Autores $^{(1)}$} \\
\cline { 2 - 6 } & 1 & 2 & 3 & 4 & 5 & 6 \\
\hline $\mathrm{N}(\mathrm{dag} / \mathrm{kg})$ & $2,60-3,40$ & $2,50-3,00$ & $2,70-3,20$ & $2,30-3,00$ & $2,90-3,20$ & $3,00-3,50$ \\
$\mathrm{P}(\mathrm{dag} / \mathrm{kg})$ & $0,15-0,20$ & $0,15-0,20$ & $0,15-0,20$ & $0,12-0,20$ & $0,16-0,19$ & $0,12-0,20$ \\
$\mathrm{~K}(\mathrm{dag} / \mathrm{kg})$ & $2,10-2,50$ & $2,10-2,60$ & $1,90-2,40$ & $2,00-2,50$ & $2,20-2,50$ & $1,80-2,50$ \\
$\mathrm{Ca}(\mathrm{dag} / \mathrm{kg})$ & $0,75-1,50$ & $0,75-1,50$ & $1,00-1,40$ & $1,00-2,50$ & $1,30-1,50$ & $1,00-1,50$ \\
$\mathrm{Mg}(\mathrm{dag} / \mathrm{kg})$ & $0,25-0,40$ & $0,25-0,40$ & $0,31-0,36$ & $0,25-0,40$ & $0,40-0,45$ & $0,35-0,50$ \\
$\mathrm{~S}(\mathrm{dag} / \mathrm{kg})$ & $0,15-0,25$ & $0,02-0,10$ & $0,15-0,20$ & $0,10-0,20$ & $0,15-0,20$ & $0,15-0,20$ \\
$\mathrm{Cu}(\mathrm{mg} / \mathrm{kg})$ & $7-20$ & $16-20$ & $8-16$ & $10-25$ & $11-14$ & $10-50$ \\
$\mathrm{Fe}(\mathrm{mg} / \mathrm{kg})$ & $70-200$ & $70-200$ & $90-180$ & $70-125$ & $100-130$ & $100-200$ \\
$\mathrm{Zn}(\mathrm{mg} / \mathrm{kg})$ & $15-30$ & $15-30$ & $8-16$ & $12-30$ & $15-20$ & $10-20$ \\
$\mathrm{Mn}(\mathrm{mg} / \mathrm{kg})$ & $50-100$ & $50-100$ & $120-210$ & $50-200$ & $80-100$ & $50-100$ \\
$\mathrm{~B}(\mathrm{mg} / \mathrm{kg})$ & $40-90$ & $40-100$ & $59-80$ & $40-75$ & $50-60$ & $40-80$ \\
\hline
\end{tabular}

(1)1: Willson (1985); 2: Reuter \& Robinson (1988); 3: Malavolta (1993); 4: Mills \& Jones Junior (1996); 5: Malavolta et al. (1997); 6: Matiello (1997). 
cia no de baixa produção, ocorrendo o inverso com o zinco. No ano de baixa produção, as lavouras de baixa produtividade também apresentaram problemas com K, Ca e S, com predomínio das deficiências (Figura 2).

Tanto em Guaxupé como em São Sebastião do Paraíso foram amostradas apenas duas lavouras com produtividade inferior a $900 \mathrm{~kg} /$ ha de café beneficia- do na média de dois anos. Por essa razão, realizou-se o diagnóstico dividindo as lavouras em duas faixas de produtividade, alta e média mais baixa. As lavouras de alta produtividade, no ano de alta produção, apresentaram problemas nutricionais de $\mathrm{Mn}, \mathrm{Cu}, \mathrm{B}$ e $\mathrm{Mg}$, e no ano de baixa produção, de $\mathrm{Mn}, \mathrm{Cu}$ e S (Figura 3). O Mn apresentou deficiência no ano de alta carga e excesso no ano de baixa carga. $\mathrm{O} \mathrm{Cu}$ apre-

Lavouras de alta produtividade
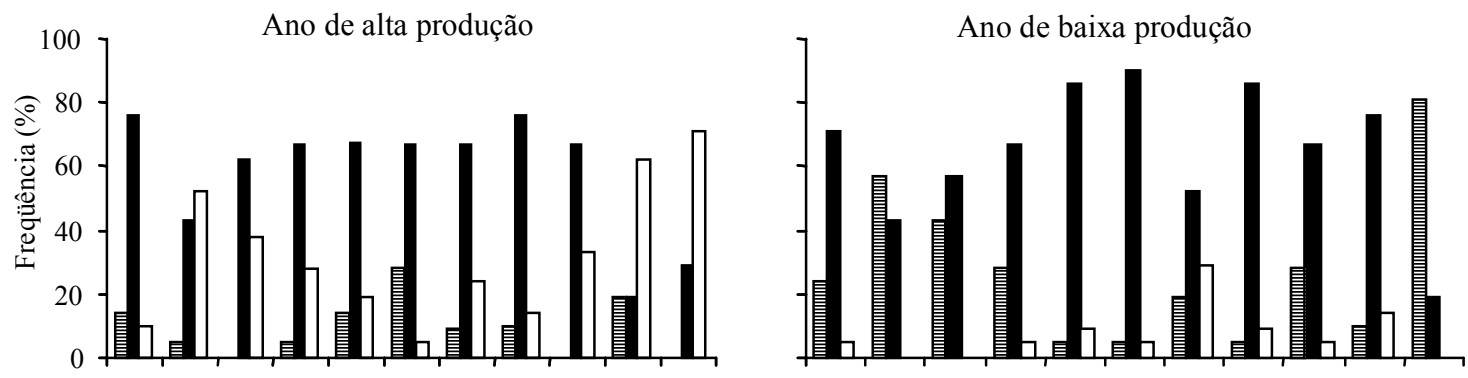

Lavouras de média produtividade
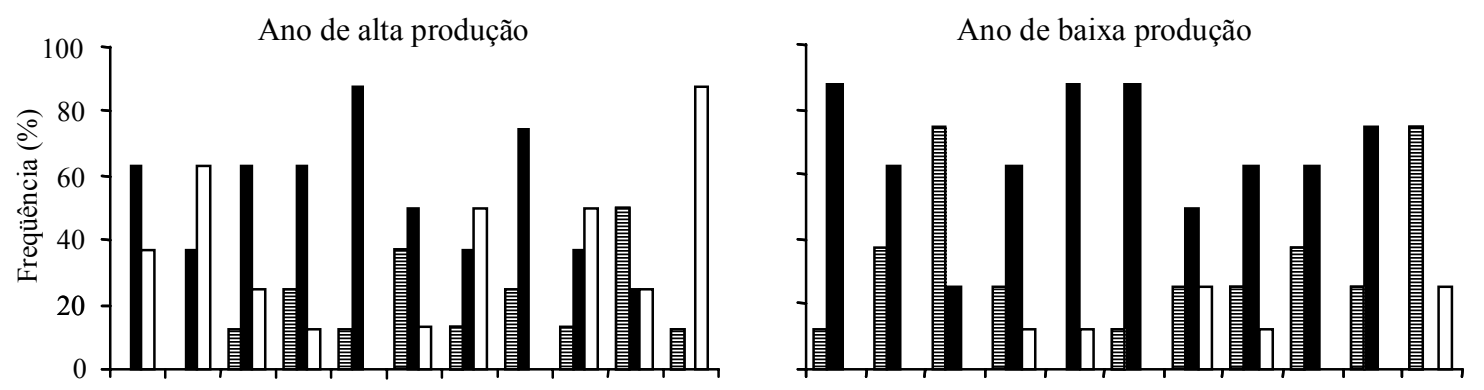

Lavouras de baixa produtividade
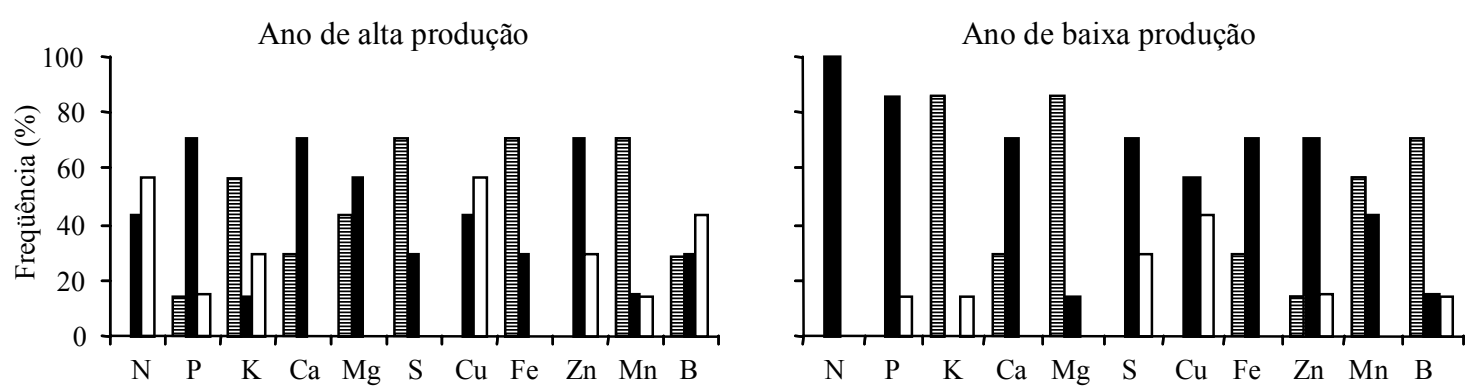

Figura 1. Freqüência de lavouras de alta, de média e de baixa produtividade da região de Manhuaçu de acordo com as classes de concentrações foliares de macronutrientes e micronutrientes (트: excessivo, $\mathbf{\square}:$ adequado, $\square$ : deficiente), em ano de alta e de baixa produção. 
sentou deficiências e excessos em ambos os anos e o B, deficiência e excesso no ano de alta e apenas excesso no ano de baixa carga. No ano de produção alta, o Mg foi deficiente em 39\% das lavouras; no de baixa, 33\% das lavouras foram deficientes em S (Figura 3).

A análise desses resultados e as informações obtidas nos questionários aplicados mostram que, provavelmente, no ano de alta carga pendente aplicaram-se quantidades excessivas de corretivos, o que levou ao aparecimento de deficiência de $\mathrm{Mn}$, micronutriente cuja disponibilidade na solução do solo é determinada pelo $\mathrm{pH}$ e pelo potencial redox, podendo sofrer restrição acentuada em condições de aeração adequada e pH elevado. É provável, ainda, que se tenha usado calcário com baixas concen-

Lavouras de alta produtividade
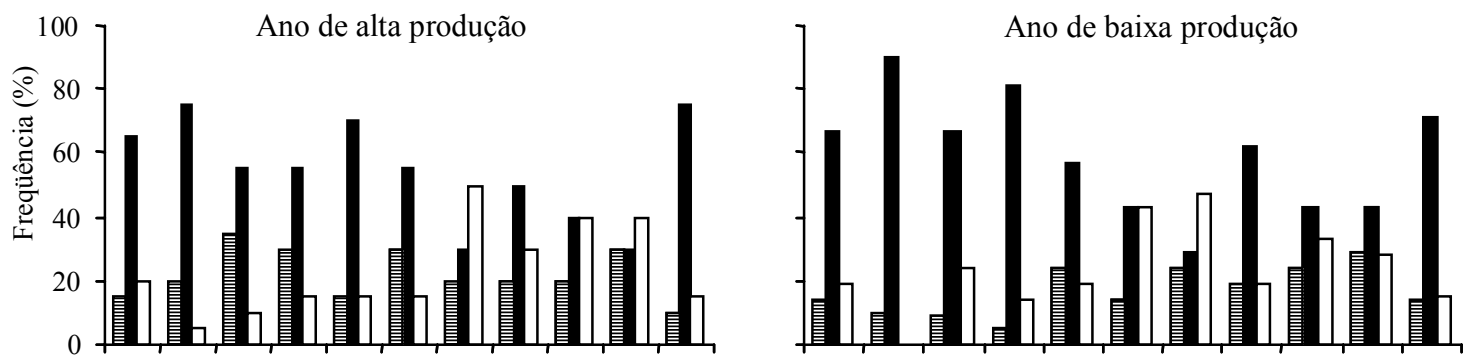

Lavouras de média produtividade
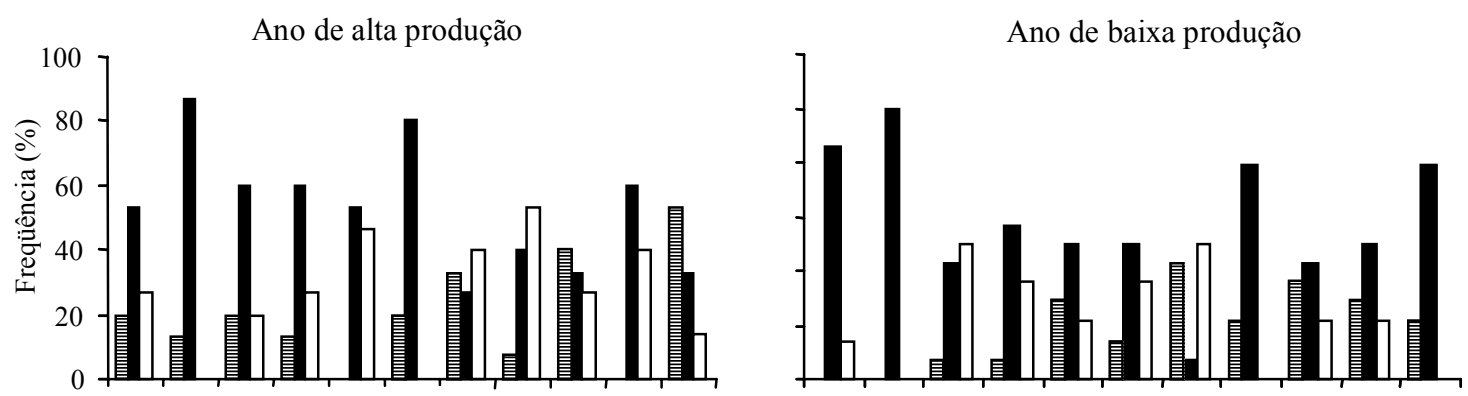

Lavouras de baixa produtividade
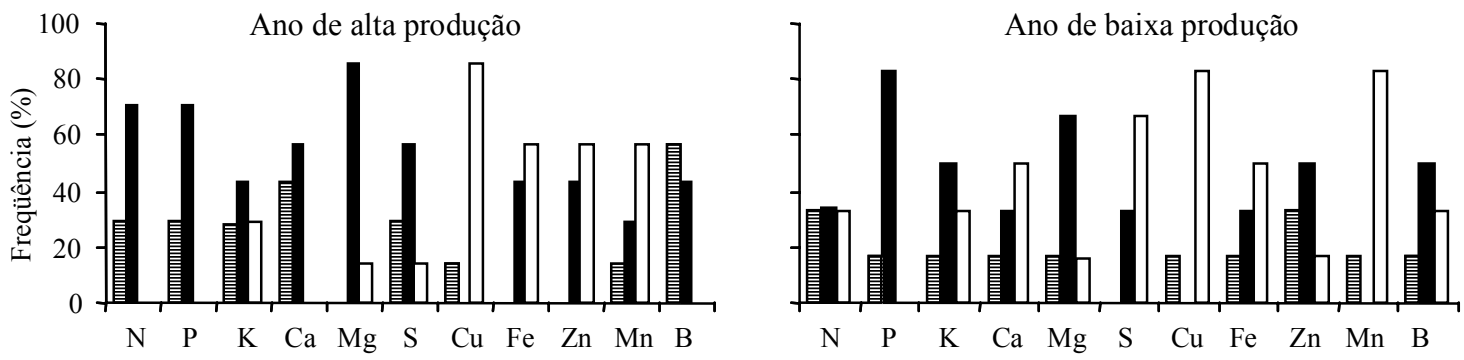

Figura 2. Freqüência de lavouras de alta, de média e de baixa produtividade da região de Patrocínio de acordo com as classes de concentrações foliares de macronutrientes e micronutrientes (豆: excessivo, $\mathbf{\square}$ : adequado, $\square$ : deficiente), em ano de alta e de baixa produção. 
trações de $\mathrm{Mg}$, ou adubações com doses elevadas de $\mathrm{K}$, o que resultou em carência desse elemento em algumas situações. No ano subseqüente, a situação quanto ao $\mathrm{Mn}$ se inverteu, com maior porcentual de lavouras com excesso do que com carência. Esse comportamento pode derivar das altas doses de adubos nitrogenados empregadas no ano anterior, em que a produtividade foi elevada, as quais podem ter promovido a acidificação dos solos, aumentando a disponibilidade de manganês. Carências de $\mathrm{S}$ no ano de baixa produção podem estar associadas às doses mais baixas de adubo nitrogenado (sulfato de amônio) empregadas nessas situações.

As lavouras de produtividade média e baixa de Guaxupé e São Sebastião do Paraíso apresentaram problemas quanto ao $\mathrm{Mn}, \mathrm{B}, \mathrm{Cu}, \mathrm{N}$ e $\mathrm{Mg}$ no ano de alta produção, e quanto ao $\mathrm{Mg}, \mathrm{S}$ e $\mathrm{Mn}$ no ano de baixa. No ano de alta produção predominaram as deficiências, de $\mathrm{N}$ e $\mathrm{Mg}$ e excesso de $\mathrm{Cu}$; quanto ao $\mathrm{B}$, excesso e deficiência ocorreram em igual propor- ção. Houve maior proporção de lavouras deficientes de Mn em ambos os anos. No ano de baixa produção ocorreram ainda deficiência de $\mathrm{Mg}$ e excesso de $\mathrm{S}$ (Figura 3).

Em Viçosa, a maioria das lavouras de alta produtividade apresentou concentrações foliares de nutrientes dentro das faixas críticas calculadas para a região, tanto no ano de alta como no de baixa produção (Figura 4). Nas lavouras de produtividade média verificaram-se, em sua maioria, concentrações fora da faixa considerada adequada de $\mathrm{B}$ e Fe, tanto no ano de alta como no de baixa produção. Concentrações excessivas e insuficientes de B apareceram em proporções semelhantes no ano de baixa produção; quanto ao $\mathrm{Fe}$, houve maior proporção de carência do que de excesso em ambos os anos. Além das concentrações inadequadas de Fe e B, no ano de baixa produção, detectaram-se lavouras com concentrações foliares de Mg excessivas e deficientes (Figura 4).

Lavouras de alta produtividade
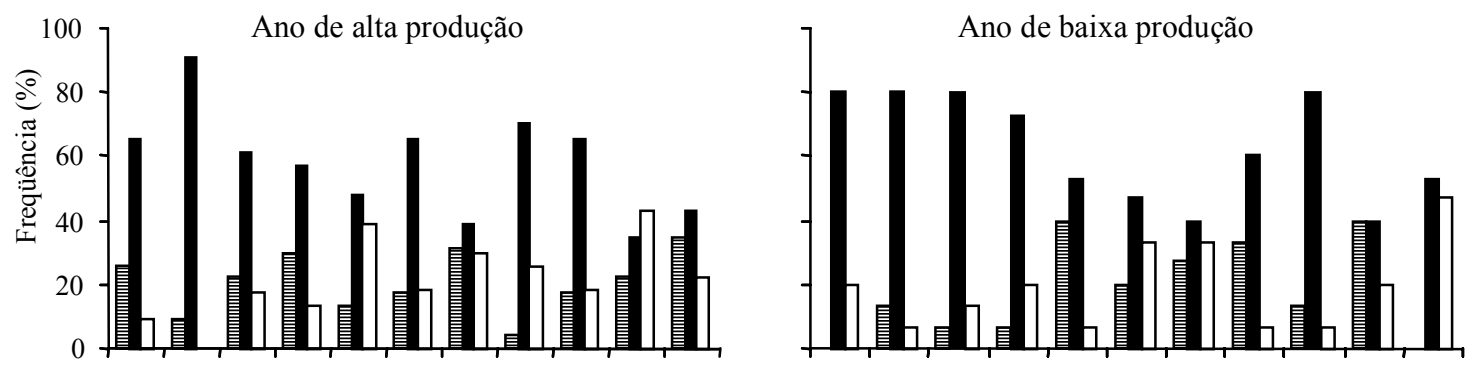

Lavouras de média e baixa produtividade
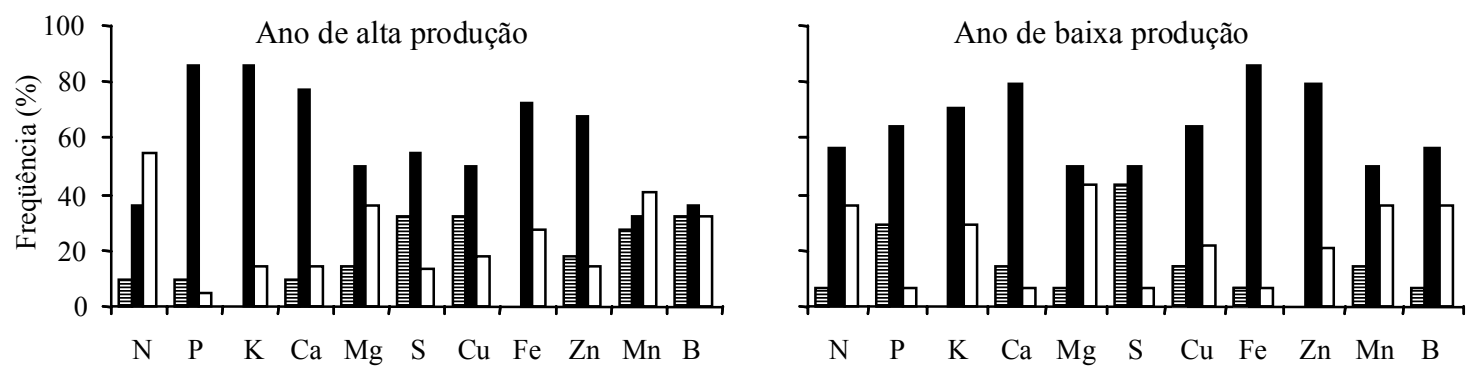

Figura 3. Freqüência de lavouras de alta e de média mais baixa produtividade nas regiões de Guaxupé e São Sebastião do Paraíso de acordo com as classes de concentrações foliares de macronutrientes e micronutrientes (ㅌ: excessivo, $\mathbf{\square}$ : adequado, $\square$ : deficiente), em ano de alta e de baixa produção. 
As lavouras de baixa produtividade da região de Viçosa apresentaram deficiência acentuada de K em ambos os anos, mais generalizada no ano de alta produção. No ano de baixa produção, a carência de $\mathrm{K}$ provavelmente restringiu a manifestação de outras carências, uma vez que, após o N, é o nutriente mais exigido quantitativamente pelos cafeeiros (Malavolta, 1993). Já no ano de alta produção, cuja demanda por nutrientes é maior, o porcentual de lavouras deficientes em $\mathrm{K}$ foi também maior, manifestando-se ainda deficiências e excessos de B e Mn nessas lavouras.

Em todas as localidades há mais problemas com relação aos micronutrientes (Figuras 1 a 4). Com exceção de Manhuaçu e São Sebastião do Paraíso, as lavouras menos produtivas apresentaram problemas

Lavouras de alta produtividade
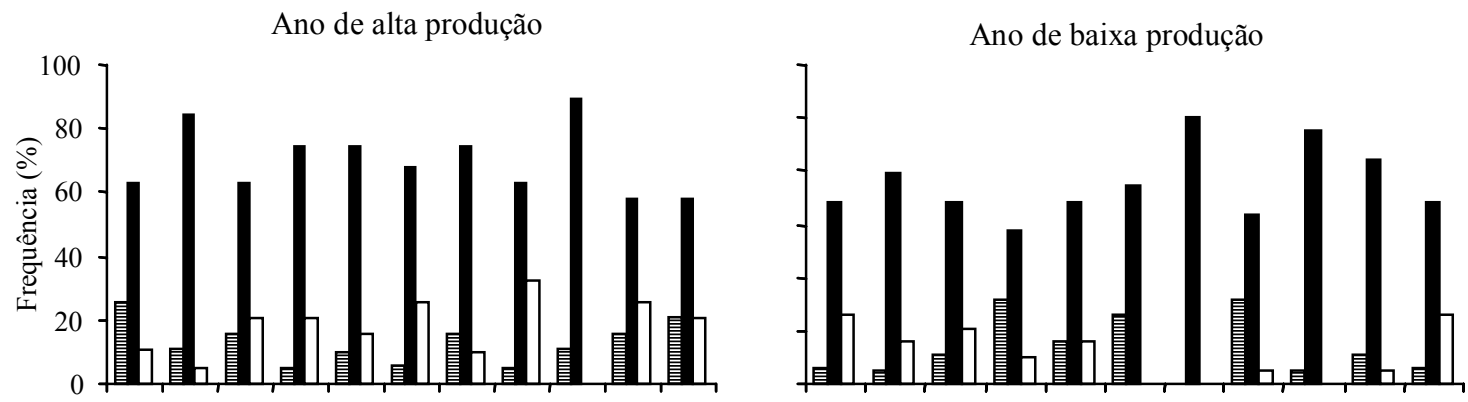

Lavouras de média produtividade
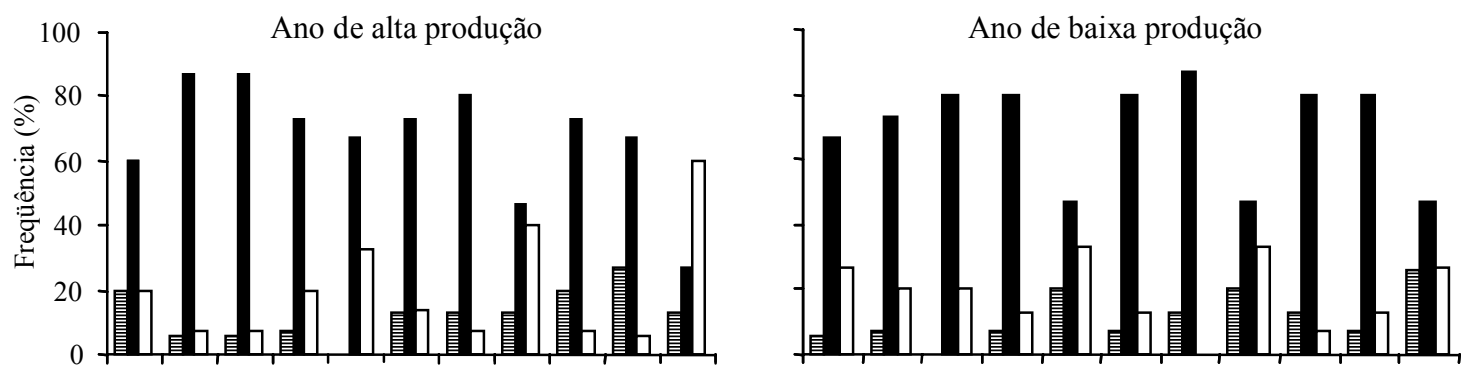

Lavouras de baixa produtividade
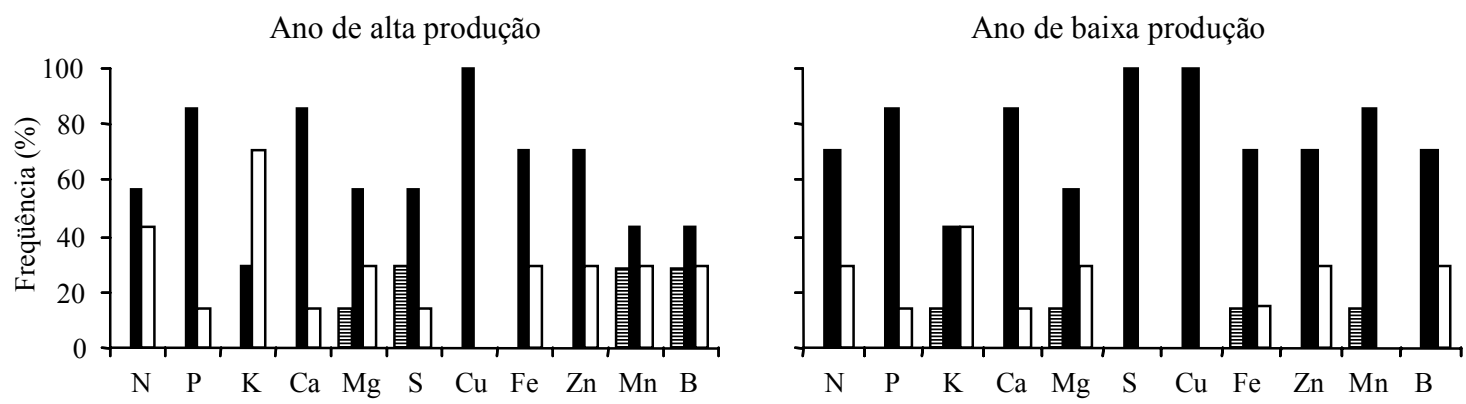

Figura 4. Freqüência de lavouras de alta, de média e de baixa produtividade da região de Viçosa de acordo com as classes de concentrações foliares de macronutrientes e micronutrientes (르: excessivo, $\mathbf{\square}$ : adequado, $\square$ : deficiente), em ano de alta e de baixa produção. 
nutricionais mais acentuados. Acredita-se que nesta região restrições acentuadas ao crescimento e produção devidas à falta de macronutrientes, como o $\mathrm{N}$, por exemplo, podem ter impedido a manifestação de outras carências. Isso pode indicar, ainda, que outros fatores além do estado nutricional estariam restringindo a produção, como arquitetura da planta e sanidade. É certo que carências ou excessos de nutrientes podem promover o depauperamento das plantas, causando morte de gemas apicais, morte descendente de ramos e brotação de ramos ladrões. Em tais condições, a capacidade produtiva é perdi$\mathrm{da}$, ainda que as concentrações foliares de nutrientes sejam recuperadas em anos subseqüentes. Há que se ressaltar também que a freqüência de lavouras com concentrações foliares fora da faixa crítica nada informa sobre o grau de deficiência ou excesso em cada situação.

\section{Conclusões}

1. As faixas críticas das concentrações de nutrientes em folhas de cafeeiro são semelhantes entre as diferentes regiões estudadas e na mesma região, independentemente do ciclo bianual de produtividade.

2. A situação nutricional das lavouras cafeeiras difere de acordo com a região e com o ano amostrado, com problemas nutricionais mais acentuados nas lavouras menos produtivas.

3. Maiores desequilíbrios são observados em relação aos micronutrientes e no ano de alta produção.

\section{Referências}

BALDOCK, J. O.; SCHULTE, E. E. Plant analysis with standardized scores combines DRIS and sufficiency range approaches for corn. Agronomy Journal, Madison, v. 88, p. 448-456, 1996.

DROSSOPOULOS, B.; KOUCHAJI, G. G.; BOURANIS, D. L. Seasonal dynamics of mineral nutrients and carbohydrates by walnut tree leaves. Journal of Plant Nutrition, New York, v. 19, n. 3, p. 493-516, 1996.

INGESTAD, T.; AGREN, G. I. Plant nutrition and growth: basic principles. Plant and Soil, Dordrecht, v. 168/169, p. 15-20, 1995.

JONES JUNIOR, J. B.; WOLF, B.; MILLS, H. A. Plant analysis handbook: a practical sampling, preparation, analysis and interpretation guide. Athens, Estados Unidos: Micro-Macro, 1991. 213 p.

KOTUR, S. C.; SINGH, H. P. Leaf-sampling technique in litchi (Litchi sinensis). Indian Journal of Agricultural Sciences, New Delhi, v. 63, n. 10, p. 632-638, 1993.

LEECE, D. R.; ENDE, B. van den. Diagnostic leaf analysis for stone fruit. Australian Journal of Experimental Agriculture and Animal Husbandry, Collingwood, v. 15, n. 72 , p. $123-128,1975$

LOOMIS, W. D.; DURST, R. W. Chemistry and biology of boron. Biofactors, Oxford, v. 3, n. 4, p. 229-239, 1992.

LUCENA, J. J. Methods of diagnosis of mineral nutrition of plants: a critical review. Acta Horticulturae, Leuven, v. 448, p. 179-192, 1997.

MALAVOLTA, E. Nutrição mineral e adubação do cafeeiro: colheitas econômicas máximas. São Paulo: Agronômica Ceres, 1993. 210 p.

MALAVOLTA, E.; CRUZ, V. F. A meaning for foliar diagnosis. In: SAMISH, R. M. (Ed.). Recent advances in plant nutrition. New York: Gordon \& Breach Science, 1971. v. 1.

MALAVOLTA, E.; VITTI, G. C.; OLIVEIRA, S. A. Avaliação do estado nutricional das plantas: princípios e aplicações. 2. ed. Piracicaba: Associação Brasileira para Pesquisa do Fosfato, 1997. 238 p.

MARTINEZ, H. E. P.; CARVALHO, J. G.; SOUZA, R. B. Diagnose foliar. In: RIBEIRO, A. C.; GUIMARÃES, P. T. G.; ALVAREZ VENEGAS, V. H. (Ed.). Recomendações para uso de corretivos e fertilizantes em Minas Gerais: 5a aproximação. Viçosa, MG: Comissão de Fertilidade do Solo do Estado de Minas Gerais, 1999 p. $143-168$.

MARTINEZ, H. E. P.; SOUZA, R. B.; ALVAREZ VENEGAS, V. H.; MENEZES, J. F. S.; OLIVEIRA, J. A.; GUIMARÃES, P. T. G.; ALVARENGA, A. de P.; FONTES, P. C. R. Avaliação da fertilidade do solo, padrões para diagnose foliar e potencial de resposta à adubação de lavouras cafeeiras de Minas Gerais. In: ZAMBOLIM, L. Café: produtividade, qualidade e sustentabilidade. Viçosa, MG: Laércio Zambolim, 2000, p. 209-238.

MATIELLO, J. B. Gosto do meu cafezal. Rio de Janeiro: Globo, 1997. 139 p.

MILLS, H. A.; JONES JUNIOR, J. B. Plant analysis handbook II. $2^{\text {nd }}$ ed. Athens: Micro-Macro, 1996. 422 p. 
OLIVEIRA, P. L. C.; ALVES. R. M. L. Certificação da cadeia produtiva do café. Revista Alimentação/Negócios do Estadão, São Paulo, v. 38, p. 76-78, 2001.

REUTER, D. J.; ROBINSON, J. B. Plant analysis: an interpretation manual. Melbourne: Inkata, 1988. 218 p.

SMITH, F. W. Interpretation of plant analysis: concepts and principles. In: REUTER, D. J.; ROBSON, J. B. (Ed.). Plant analysis: an interpretation manual. Melbourne: Inkata, 1988. p. 1-12.
SOLTAMPOUR, P. N.; MALAKOUTI, M. J.; RONAGHI, H. Comparison of diagnosis and recommendation integrated system and nutrient sufficiency range for corn. Soil Science Society of America Journal, Madison, v. 59, p. 133-139, 1995.

WILLSON, K. C. Mineral nutrition and fertilizer needs. In: CLIFORD, N. N.; WILLSON, K. C. (Ed.). Coffee botany, biochemistry and production of beans and beverage. London: Croom Helm, 1985. p. 135-156. 